\title{
Analysis of space-time coupling in SEA-SPIDER measurements
}

\author{
Adam S. Wyatt and Ian A. Walmsley \\ Clarendon Laboratory, University of Oxford, Parks Road, Oxford, OX1 3PU, UK
}

Space-time coupling (STC) is a well known phenomenon of ultrashort pulses in which the electric field of pulse can not be factorized into separate complex functions of space and time. STC is extremely important in ultrafast optics as it allows control of dispersion and is necessary for pulse shaping. Measuring STC easily and accurately allows one to ensure they have obtained the optimal pulse for their experiment. In addition, utilizing STC in nonlinear processes may enable one to fine-tune the control of the dynamics of the process.

Pulse characterisation methods based on non-collinear frequency mixing (NCFM) can introduce additional STC in the measurement. Thus it is necessary to re-examine the NCFM process in order to be able to measure STC accurately with these devices. SEA-SPIDER (fig. 1 (a) illustrates the concept), is one characterisation method that in principle can measure the temporal electric field of a pulse as a function of a single spatial dimension, thus measuring STC (but not the spatial phase) $[1,2]$. We have modelled this method specifically and found it is necessary to account for this additional 'geometric' STC for few-cycle pulses.

In SPIDER, it is necessary to ensure that the test pulse (TP) upconverts with a single quasimonochromatic frequency. In order to measure STC, it is necessary to ensure that the ancilla pulse is a single plane wave over the spatial extent of the TP as well, otherwise this can introduce additional STC to the signal pulse (SP). This can be ensured by spatially filtering the ancilla. We have studied numerous geometries for performing the spatial filtering and discuss the merits of each.

In addition, the non-collinear geometry results in additional angular dispersion on the measured pulse, even if the TP was initially factorizable in space and time. This has been shown schematically by the phase matching diagrams in fig. 1 (b) and (c). As the fringe spacing of the interferogram (fig. 1 (d) shows an example) is given by $\left(\mathbf{k}_{\mathbf{1}}-\mathbf{k}_{\mathbf{2}}\right)$. $\mathbf{x}$, the fringe periodicity for zero shear is independent of frequency. Compare this to spectral interferometry, where the fringe periodicity is proportional to frequency.
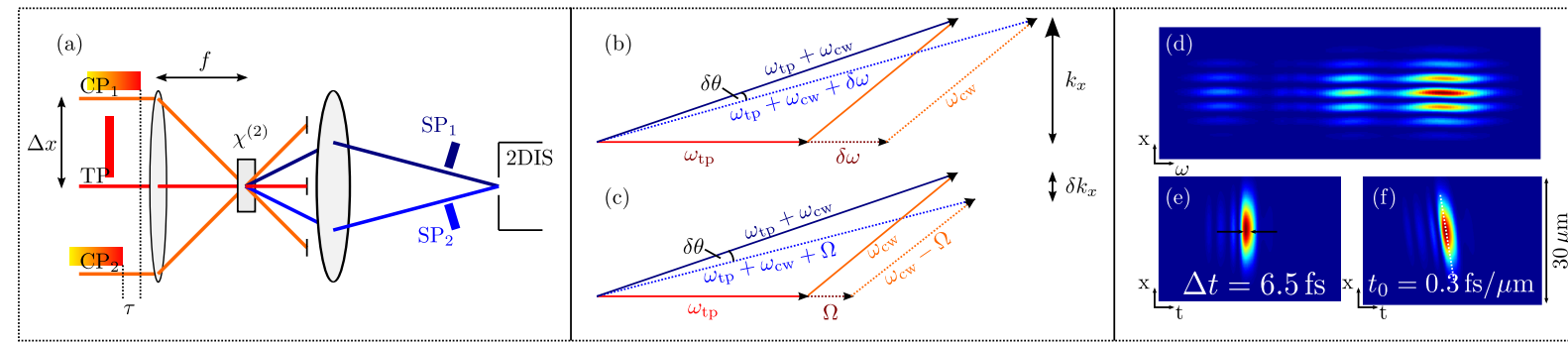

Fig. 1: (a) SEA-SPIDER concept. (b) Phase matching for two separate frequencies from the TP and a single frequency from the CP. (c) Phase matching for a two different TP + CP frequencies resulting in the same output frequency (i.e. $\omega_{\mathrm{TP}}+\omega_{C P}=$ const). (d) Simulated SEA-SPIDER interferogram. (e) Space-time intensity of the TP. (f) Simulated effect of extra STC from the SFG.

Since the carrier fringe periodicity depends on the ancilla frequency, the angular dispersion of the measured pulse will have an additional term given by $E_{\text {meas }}(\omega, x)=E_{\mathrm{TP}}(\omega, x) \exp [i(\Delta x / f c) \omega x]$, where the parameters are defined in 1 (a) and $x$ is the spatial co-ordinate across the beam in the crystal. Using typical values of $\Delta x=1 \mathrm{~cm}$ and $f=10 \mathrm{~cm}$, the pulse front tilt is approximately $0.3 \mathrm{fs} / \mu \mathrm{m}$, giving a total delay of several fs across the beam. This represents a small but significant number for a few-cycle pulse.

SEA-SPIDER has already been demonstrated as an excellent candidate for measuring extremely large bandwidth pulses. In addition, it has been shown that it is possible to measure the STC of a pulse in one spatial dimension. However, the non-collinear sum-frequency generation used in the device introduces a small amount of additional STC coupling which can easily be corrected for. We believe that this analysis will prove invaluable as it allows one to obtain more complete and accurate information about their pulses. The ideas and analysis presented here can also be applied to other characterisation methods that claim to be able to measure STC.

\section{References}

[1] E. M. Kosik, A. S. Radunsky, I. A. Walmsley, and C. Dorrer. Optics Letters, 30(3):326-328, February 2005.

[2] A. S. Wyatt, I. A. Walmsley, G. Stibenz, and G. Steinmeyer. Optics Letters, 31(12):1914-1916, June 2006. 